\title{
Quantifying Stranded Implant Displacement Following Prostate Brachytherapy
}

\author{
Julio Lobo ${ }^{1}$, Mehdi Moradi ${ }^{1}$, Nick Chng $^{2}$, Ehsan Dehghan ${ }^{3}$, \\ Gabor Fichtinger ${ }^{3}$, William J. Morris ${ }^{2}$, and Septimiu E. Salcudean ${ }^{1}$ \\ 1 Department of Electrical and Computer Engineering, \\ University of British Columbia, Vancouver, Canada \\ \{juliol, moradi, tims\}@ece.ubc.ca \\ ${ }_{2}$ British Columbia Cancer Agency \\ 3 School of Computing, Queen's University
}

\begin{abstract}
We aim to compute radioactive stranded-implant displacement during and after prostate brachytherapy. We present the methods used to identify corresponding seeds in planned, intra-operative and postimplant patient data that enable us to compute seed displacements. A minimum cost network flow algorithm is used, on 8 patients, for needle track detection to group seeds into needles that can be matched between datasets. An iterative best line detection algorithm is used both to help with needle detection and to register the different datasets. Our results show that there was an average seed misplacement of $5.08 \pm 2.35 \mathrm{~mm}$ during the procedure, which then moved another $3.10 \pm 1.91 \mathrm{~mm}$ by the time the quality assurance CT was taken. Several directional trends in different regions of the prostate were noted and commented on.
\end{abstract}

\section{Introduction}

Prostate brachytherapy is an effective, minimally invasive treatment technique for men with prostate cancer [1]. It involves transperineal insertion of permanent radioactive sources, or seeds, into the prostate using needles. Many research groups have recognized the importance of monitoring the seed displacement and have used manual seed labeling, contouring or fiducial markers [2 5] to do this. We present a novel approach to automatically register different datasets and match corresponding seeds for displacement analysis.

This paper describes the computation of the misplacement, due to prostate rotation and needle deflection, of individual seeds in a stranded implant by comparing the pre-operation planned placement (pre-plan data) to the actual placement during surgery. The latter seed positions are computed from intraoperative fluoroscopic data [6]. We extended the study to include the quantification of seed movement from the intra-operative conditions to immediately after surgery where CT reconstruction is used to compute seed positions [7]. This movement is caused by change in patient pose from dorsal lithotomy during the procedure to horizontal recumbent during CT imaging and also from immediate inflammation. We wish to point out that although the term misplacement

G. Fichtinger, A. Martel, and T. Peters (Eds.): MICCAI 2011, Part I, LNCS 6891, pp. 307-314, 2011. (C) Springer-Verlag Berlin Heidelberg 2011 


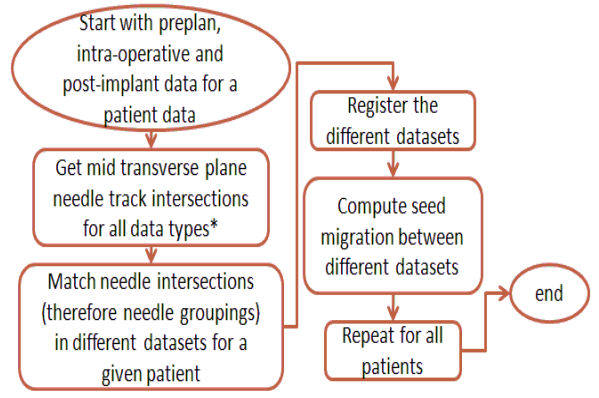

Fig. 1. Flow chart of the full process used to compute seed displacement. ( ${ }^{*}$ Figure 2 shows how the needle intersections are found).

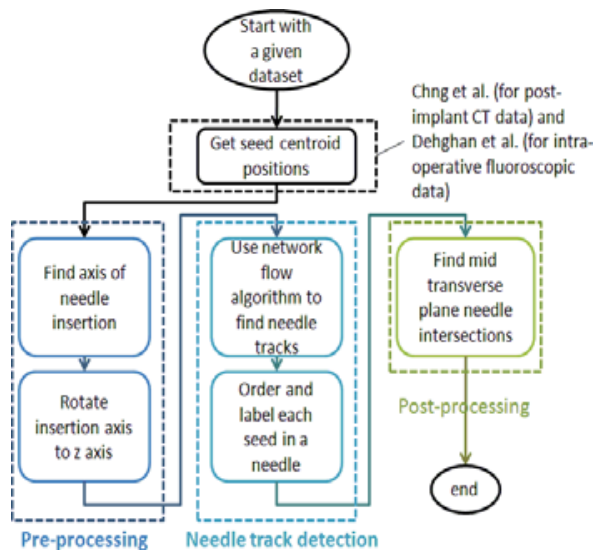

Fig. 2. Needle detection and calculation of mid transverse plane needle track intersections

may imply it, we cannot distinguish between tissue deformation and implant misplacement - analyzing this would require a separate study.

The entire process requires matching of individual seeds between these datasets to calculate the amounts of displacement. Seeds were grouped into their respective needles using a minimum cost network flow algorithm [8] (used previously by Chng et al. on CT data 7]). Needles were then matched so that individual seeds could be identified between datasets. To correctly compute the displacement of a given seed, a registration process was needed to put the pre-plan, intra-operative fluoroscopic and post-implant CT data into correspondence. A technical novelty for this process involved an iterative sampling algorithm to average potential needles and determine the implantation axis direction, which is not consistent between datasets.

Seed movement from pre-plan to intra-operative to post-implant have not been presented before. Seed displacement results will be presented after a description of the the methods used to label seeds in different datasets.

\section{Methods}

For stranded implants, seeds from the same needle stay ordered and somewhat aligned in a needle track, therefore a needle track is an intuitive class to group seeds into. Matching needle groupings between datasets simplifies the problem from matching $\approx 100$ seeds to $\approx 20$ needles and allows individual seed identification and therefore displacements computation.

The methods are summarized in Figure 1 and described in the next subsections. The needle detection process alone is summarized in Figure 2 ,

All the data used was obtained from eight consenting patients undergoing low-dose prostate brachytherapy with institutional research ethics approval. 


\subsection{Inter-dataset Seed Matching}

Detection of the Insertion Axis. We use an iterative best line detection to find the axis of insertion of needles. Our algorithm is similar to the RANSAC (RANdom SAmple Consensus, [9]) algorithm which involves fitting lines to pairs of randomly chosen points and scoring each line. The highest scored lines are then kept as needles. Lines that have large deviations from the the $\mathrm{z}$ axis, which is the cranio-caudal axis in the $\mathrm{CT}$, and the axis of rotation of the source-detector on the C-arm of the fluoroscope, are rejected immediately, saving unnecessary calculations. An exhaustive search on all possible pairs of points for this application is not computationally intensive so we have no random component. Pairs of points are used to fit straight lines and a Gaussian cone is used to cut-off seeds that are clearly not part of the needle.

Although the algorithm does not consistently assign seeds to needle groups correctly, the highest scoring lines are correct needle directions for a wide range of parameter values. The top eight needle directions, which are chosen to be consistent between datasets, were then averaged to find the implantation axis. This was not required for the pre-plan data which has the transducer axis already parallel to the $\mathrm{z}$ axis.

Needle Detection. Once the dataset is rotated so that it is aligned with the implantation axis, it is possible to use an algorithm with tighter restrictions. Because seeds from a single needle do not necessarily lie on a line, instead of looking at parameterizable lines, the seeds are viewed as nodes within a network that allow a single unit of flow through them. Each arc between two nodes carries a cost. Open source code ("matlog") written by Kay [10] was used to perform the minimization.

The cost function is made up of the same components as the scoring function iterative best line approach. Namely, these are: a measure of the angle, $\theta_{i j}$, that the arc $i j$ between seed $i$ and seed $j$ makes with the implantation axis, and a lower cost for arcs that are the length of a typical seed spacer, denoted as $s_{0}$, which is equal to integer multiples of $1 \mathrm{~cm}$. The angle and spacing costs $A C_{i j}$ and $S C_{i j}$ are defined as follows:

$$
\begin{gathered}
A C_{i j}=A\left(e^{\left|\theta_{i j}\right| / \theta_{0}}-1\right)^{4} \\
S C_{i j}=-B e^{-s_{i j} / s_{d}}\left(\left(\sum_{n=1}^{3} \delta\left(s_{i j}-n s_{0}\right)\right) *\left(e^{-s_{i j} / s_{v}}\right)^{2}\right)
\end{gathered}
$$

where $s_{i j}$ is the spacing between node $i$ and node $j$. The $*$ symbol denotes convolution. The spacing cost is defined as a Gaussian with a variance of $s_{v}$ convolved with a train of delta functions to give negative peaks at integer multiples of the smallest spacing $\left(s_{0}=10 \mathrm{~mm}\right)$. A final decaying exponential with a variance of $s_{d}$ is used to make the closer spacings more desirable. The spacing cost reflects the spacing seen in stranded implants. 
The final objective function is:

$$
C_{i j}=\gamma A C_{i j}+S C_{i j}
$$

where $\gamma$ is only used for the pre-plan data to heavily weight towards the angle.

Inter-dataset Needle Matching. After rotating the seed clusters and grouping the seeds into needles, the intersection of each needle with the most inferior transverse plane is computed. Matching is then performed using another minimum cost network flow algorithm. Each node of the network contains the x,y coordinates of an intersection as well as the number of seeds that were in the needle. Including the number of seeds per needle in each node, and the cost of a unit flow, was found to remove ambiguities caused when there are several needles close to each other.

\subsection{Cluster Registration and Seed Displacement Computation}

After the needles are matched, each seed position can be compared to its corresponding seed from a different data type to obtain a displacement vector. To do this the seeds clusters have to be registered so that they are in the same coordinate system. This registration was performed by translating each seed cluster so that the origin is at the centroid of all the seeds and then rotating it so that the $\mathrm{z}$ axis is aligned with the implantation axis. This process ensures that the centers of mass of the seed clusters are in the same place and that both the pitch and yaw of the clusters are matched. It is assumed that there is no significant differences in the rotation about the implantation axis between clusters since the bed is horizontal for both the CT and the fluoroscopic images.

An average distance was computed over all seeds from all patients for the entire volume as well as for each of the six (left, right, posterior, anterior, superior, inferior) half-sections. The average lateral, superior-inferior and anteriorposterior components of the displacement were also calculated in each section. This was done for both pre-plan to intra-operative misplacement and intraoperative to post-implant movement.

\section{$3 \quad$ Results and Discussions}

\subsection{Inter-dataset Seed Matching Results}

Iterative Best Line Detection Results. The iterative best line detection algorithm correctly assigned $79.0 \%$ to $93.2 \%$ of the seeds. $80 \%$ to $100 \%$ of the needle vectors were correctly found. In all the patients and data types, the top 8 needle directions found were correctly so the average direction gave the correct insertion axis direction. 

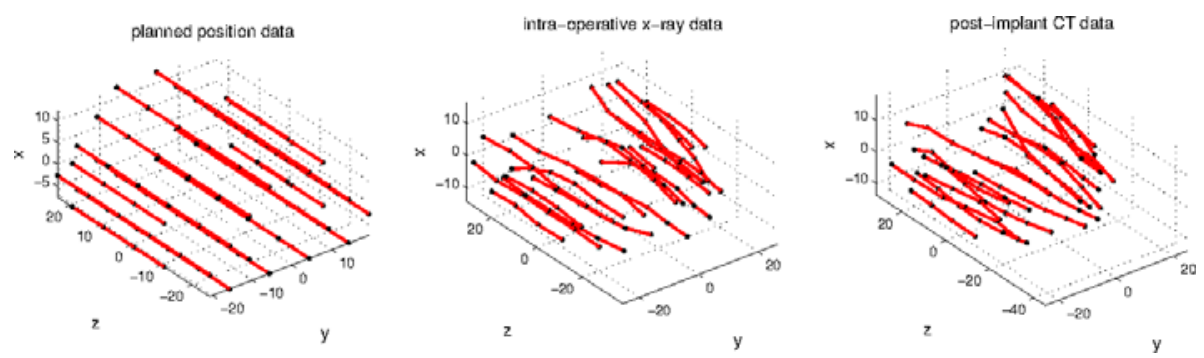

Fig. 3. Needle detection results for 1 patient for pre-plan, intra-operative and postimplant data

Minimum Cost Network Flow Needle Detection Results. Both cluster rotation and network flow calculation were tested on our 8 datasets. Figure 3 shows the results from a single patient for all three data types.

Summarizing the results, an average of $99.3 \%$ of the seeds were correctly assigned for the intra-operative data, while an average of $99.4 \%$ of the seeds were correctly assigned for the post-implant data. Once again, no pre-plan information is used in the needle detection and the algorithm takes between 1 to 2.5 seconds to rotate the cluster and find the needles. The needle detection for the pre-plan data correctly assigned all the seeds in all patients.

Inter-dataset Needle Matching Results. The few incorrectly assigned seeds in the various datasets were manually adjusted to be grouped with the correct needle. The method described earlier, achieved a correct needle matching for all the datasets, for both the pre-plan to intra-operative and intra-operative to post-implant matching.

\subsection{Seed Displacement Results}

With the needles correctly identified and matched in corresponding data sets for a given patient, the seed locations could be directly compared which gave us a measure of the seed displacement. As an example of a pre-plan to intraoperative comparison, Figure 4 shows the misplacement of the seeds for a single patient. Similar illustrations were computed for intra-operative to post-implant comparisons. Figure 5 shows all three sets of data for a single needle.

Average Displacement Magnitudes. The distance that every seed moved between datasets was computed for all 871 seeds from the 8 patient data sets, then averaged. The average displacement was significantly larger for the preplan to intra-operative case than for the intra-operative to post-implant data ( $5.08 \mathrm{~mm}$ versus $3.10 \mathrm{~mm}$ with $\mathrm{p}<0.01, \mathrm{n}=871)$ and both distances were within the reported localization error of $0.9 \mathrm{~mm}[\underline{6}]$.

The average distance was computed for each half-section in the 3D space to quantify the motion in each region. Table 1 shows these distances. 


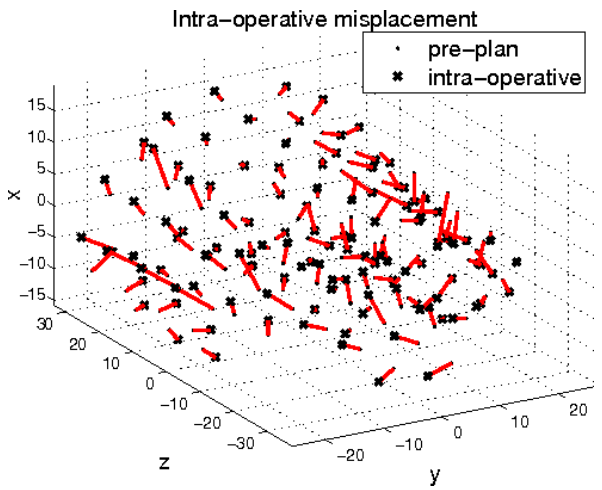

Fig. 4. Pre-plan to intra-operative seed misplacement results

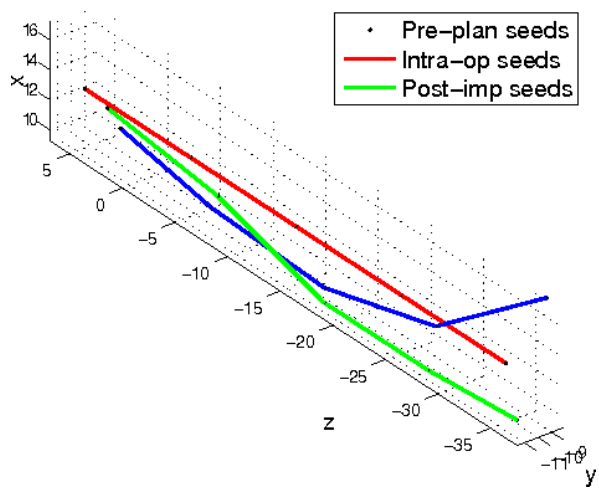

Fig. 5. Seed positions of a single needle from all three datasets

Table 1. Seed displacement results for different regions within the prostate volume

\begin{tabular}{|c|c|c|}
\hline Half-section & $\begin{array}{c}\text { Av. misplacement } \\
\text { (pre to intra) } / \mathrm{mm}\end{array}$ & $\begin{array}{c}\text { Av. movement } \\
\text { (intra to post) } / \mathrm{mm}\end{array}$ \\
\hline \hline left & $5.07 \pm 2.46$ & $3.11 \pm 1.87$ \\
right & $5.15 \pm 2.43$ & $3.12 \pm 1.78$ \\
\hline inferior & $4.94 \pm 2.47$ & $3.19 \pm 1.87$ \\
superior & $5.29 \pm 2.42$ & $3.04 \pm 1.78$ \\
\hline posterior & $4.63 \pm 2.34$ & $2.97 \pm 1.61$ \\
anterior & $5.60 \pm 2.55$ & $3.26 \pm 2.04$ \\
\hline
\end{tabular}

Table 2. Displacement results for intra-operative misplacement and post-implant movement

\begin{tabular}{|c||c|c|c||c|c|c|}
\hline \multicolumn{1}{|c||}{} & \multicolumn{2}{c||}{ Intra-operative misplacement } & \multicolumn{2}{c|}{ Post-implant movement } \\
\hline $\begin{array}{c}\text { Half- } \\
\text { section }\end{array}$ & $\begin{array}{c}\text { lateral } \\
/ \mathrm{mm}\end{array}$ & $\begin{array}{c}\text { inf-sup } \\
/ \mathrm{mm}\end{array}$ & $\begin{array}{c}\text { ant-post } \\
/ \mathrm{mm}\end{array}$ & $\begin{array}{c}\text { lateral } \\
/ \mathrm{mm}\end{array}$ & $\begin{array}{c}\text { inf-sup } \\
/ \mathrm{mm}\end{array}$ & $\begin{array}{c}\text { ant-post } \\
/ \mathrm{mm}\end{array}$ \\
\hline \hline left & $-0.42 \pm 1.69$ & $0.13 \pm 2.71$ & $0.28 \pm 1.81$ & $0.34 \pm 0.94$ & $-0.32 \pm 1.63$ & $0.09 \pm 1.18$ \\
right & $0.44 \pm 1.93$ & $-0.31 \pm 2.28$ & $-0.20 \pm 1.85$ & $-0.28 \pm 1.00$ & $0.29 \pm 2.06$ & $0.08 \pm 1.16$ \\
\hline inf & $-0.22 \pm 1.54$ & $-0.27 \pm 2.66$ & $-0.02 \pm 1.70$ & $0.05 \pm 0.99$ & $0.28 \pm 1.83$ & $0.24 \pm 1.14$ \\
sup & $0.24 \pm 2.11$ & $0.09 \pm 2.34$ & $0.10 \pm 1.99$ & $0.01 \pm 1.05$ & $-0.31 \pm 1.89$ & $-0.07 \pm 1.18$ \\
\hline post & $0.34 \pm 1.16$ & $2.02 \pm 1.30$ & $-0.30 \pm 1.63$ & $-0.10 \pm 1.14$ & $0.40 \pm 1.55$ & $-0.13 \pm 1.24$ \\
ant & $-0.32 \pm 2.32$ & $-2.19 \pm 1.32$ & $0.38 \pm 1.98$ & $0.16 \pm 0.86$ & $-0.44 \pm 2.08$ & $0.29 \pm 1.06$ \\
\hline
\end{tabular}

For both pre-plan to intra-operative and intra-operative to post-implant displacements, there were no significant differences in the amount of motion between the different half sections. All half-sections, however, had larger pre-plan to intra-operative misplacement as compared to intra-operative to post-implant movement. 
Displacement Vectors. Table 2 summarizes the general displacement directions seen.

As with the scalar measurements, these directional displacements were smaller for the intra-operative to post-implant case than in the pre-plan to intra-operative case. With the calculated standard deviations, the only statistically significant displacement was the superior misplacement of the posterior seeds and the inferior misplacement of anterior seeds between the pre-plan and intra-operative conditions. No other significant trends were seen.

\section{Summary and Conclusions}

We have combined several techniques, including the use of an iterative best line detection algorithm and network flow algorithms, to formulate a new method to identify seeds from stranded implants in different datasets and therefore compute their displacements.

For the pre-plan to intra-operative comparison, the inferior misplacement of anterior seeds and superior misplacement of posterior seeds is in agreement with discussions with oncologists on preferred placement. Since there is a lack of implantable tissue in the anterior superior quadrant (close to the bladder), seeds are deliberately placed more inferiorly. Also, the divergence of the rectum from the prostate in the superior posterior quadrant leads to a tendency to "over-plan" this region.

The misplacement of seeds (due to needle deflection and prostate rotation) is shown to be more significant than the movement immediately after surgery. This agrees with work done by Wan et al. to evaluate needle deflection [11] and by Lagerburg et al. who evaluate prostate rotation during the insertion of needles [12]. The results also emphasize the seed placement process as a research area that requires more attention, in comparison to immediate post-implant seed movement, in future efforts to improve prostate brachytherapy.

The algorithms explained in this paper have been described for pre-plan to intra-operative fluoroscopic to post-implant CT-data. However, they can also be used to compare seed positions over several days after a surgery to further monitor inflammation. Another application of the developed algorithms is in real-time dosimetry. Complete ultrasound-based brachytherapy seed detection is a notoriously challenging problem. However, Moradi et al. have shown that given a complete reconstruction of the fluoroscopic data and identification of the seeds, registration can be performed with an incomplete reconstruction of the seed cloud in ultrasound data [13]. Therefore, the complete seed identification (or labeling) algorithm described here, could make it possible to perform intraoperative dosimetry.

Acknowledgments. The work was supported by NSERC and CIHR. Gabor Fichtinger was supported as a Cancer Care Ontario Research Chair. 


\section{References}

1. Morris, W., Keyes, M., Palma, D., Spadinger, I., McKenzie, M., Agranovich, A., Pickles, T., Liu, M., Kwan, W., Wu, J.: Population-based study of biochemical and survival outcomes after permanent 125I brachytherapy for low- and intermediaterisk prostate cancer. Urology 73(4), 860-865 (2009)

2. Roberson, P.L., Vrinda, N., Daniel, L.M., Raymond, J.W., McLaughlin, P.W.: Effects of seed migration on post-implant dosimetry of prostate brachytherapy. Medical Physics 24, 251-257 (1997)

3. Pinkawa, M., Gagel, B., Asadpour, B., Piroth, M., Klotz, J., Borchers, H., Jakse, G., Eble, M.: Seed displacements after permanent brachytherapy for prostate cancer in dependence on the prostate level. Strahlentherapie und Onkologie 184, 520525 (2008) 10.1007/s00066-008-1886-3

4. Pinkawa, M., Asadpour, B., Piroth, M.D., Gagel, B., Klotz, J., Fischedick, K., Borchers, H., Jakse, G., Eble, M.J.: Rectal dosimetry following prostate brachytherapy with stranded seeds - comparison of transrectal ultrasound intra-operative planning (day 0) and computed tomography-postplanning (day 1 vs. day 30) with special focus on sources placed close to the rectal wall. Radiotherapy and Oncology $91(2), 207-212(2009)$

5. Usmani, N., Chng, N., Spadinger, I., Morris, W.J.: Quantification of migration of 125iodine rapidstrands in prostate brachytherapy implants in different regions of the prostate. In: Genitourinary Cancers Symposium, vol. 96 (2010)

6. Dehghan, E., Lee, J., Moradi, M., Wen, X., Fichtinger, G., Salcudean, S.E.: Prostate brachytherapy seed reconstruction using C-arm rotation measurement and motion compensation. In: Jiang, T., Navab, N., Pluim, J.P.W., Viergever, M.A. (eds.) MICCAI 2010. LNCS, vol. 6361, pp. 283-290. Springer, Heidelberg (2010)

7. Chng, N., Spadinger, I., Morris, W., Usmani, N., Salcudean, S.: Prostate brachytherapy postimplant dosimetry: Automatic plan reconstruction of stranded implants. Brachytherapy (2010)

8. Ahuja, R., Magnanti, T., Orlin, J.: Network Flows: Theory, Algorithms, and Applications. Prentice Hall, Englewood Cliffs (1993)

9. Fischler, M.A., Bolles, R.C.: Random sample consensus: A paradigm for model fitting with applications to image analysis and automated cartography. Comm. of the ACM, 381-395 (1981)

10. Kay, M.G., Parlikad, A.N.: Material flow analysis of public logistics networks. Progress in Material Handling Research, 205-218 (2002)

11. Wan, G., Wei, Z., Gardi, L., Downey, D.B., Fenster, A.: Brachytherapy needle deflection evaluation and correction. Medical Physics 32(4), 902-909 (2005)

12. Lagerburg, V., Moerland, M.A., Lagendijk, J.J., Battermann, J.J.: Measurement of prostate rotation during insertion of needles for brachytherapy. Radiotherapy and Oncology 77(3), 318-323 (2005)

13. Moradi, M., Wen, X., Dehghan, E., Lobo, J., Morris, W.J., Salcudean, S.E.: Needle path detection for brachytherapy dosimetry based on lateral power imaging and template matching. In: IEEE Ultrasonics Symposium, pp. 586-589 (2009) 